\title{
Early pericalcarine atrophy in acute optic neuritis is associated with conversion to MS
}

T M Jenkins, ${ }^{1}$ O Ciccarelli, ${ }^{1}$ M Atzori, ${ }^{1}$ C A M Wheeler-Kingshott,,${ }^{1}$ D H Miller, ${ }^{2}$ A J Thompson ${ }^{1}$, A T Toosy ${ }^{1}$

1 Department of Brain Repair and Rehabilitation, UCL Institute of Neurology, Queen Square, London, WC1N 3BG, UK

2 Department of Neuroinflammation, UCL Institute of Neurology, Queen Square, London, WC1N 3BG, UK

\section{Correspondence to}

Dr. Ahmed T. Toosy,

Department of Brain Repair and Rehabilitation,

UCL Institute of Neurology,

Queen Square,

London,

WC1N 3BG,

United Kingdom;

Email: a.toosy@ion.ucl.ac.uk

Key words: optic neuritis, multiple sclerosis, pericalcarine, atrophy, magnetic resonance imaging Word count: 3159 


\begin{abstract}
Background Previous work showed that pericalcarine cortical volume loss is evident early after presentation with acute clinically isolated optic neuritis (ON). The aims of this study were: (1) To determine whether pericalcarine atrophy predicts conversion to multiple sclerosis (MS); (2) To investigate whether regional atrophy specifically affects visual cortex; (3) To investigate potential causes of early pericalcarine atrophy using magnetic resonance imaging (MRI).
\end{abstract}

Methods Twenty-eight patients with acute $\mathrm{ON}$ and ten controls underwent structural MRI (brain and optic nerves) and were followed up over 12 months. Associations between the development of MS, optic nerve, optic radiation and pericalcarine cortical damage measures were investigated using multiple regression models. Regional cortical volumetric differences between patients and controls were calculated using t-tests.

Results The development of MS at 12 months was associated with greater whole-brain and optic radiation lesion loads, shorter acute optic nerve lesions and smaller pericalcarine cortical volume at baseline. Regional atrophy was not evident in other cortical regions. Pericalcarine atrophy was not directly associated with whole-brain lesion load, optic radiation measures or optic nerve lesion length. However, the association between pericalcarine atrophy and MS was not independent of these parameters.

Conclusions Reduced pericalcarine cortical volumes in patients with early clinically isolated ON predicts the development of MS, but volumes of other cortical regions early after ON are not predictive. Hence, pericalcarine cortical regions appear particularly susceptible to early damage. These findings could be explained by a combination of pathological effects to visual grey and white matter in patients with ON. 


\section{INTRODUCTION}

In recent years, the clinical importance of grey matter damage in multiple sclerosis (MS) has become increasingly clear. We recently identified pericalcarine cortical volume loss on magnetic resonance imaging (MRI) in patients with acute, clinically isolated ON.[1] Pericalcarine cortical volume was found to be $14 \%$ smaller in ON patients than in controls $(\mathrm{p}=0.047)$. Whilst it is recognised that both generalised and regional grey matter atrophy occur early in the course of MS, [2-4] it is unknown whether regional atrophy evident at presentation is associated with conversion. It also remains unclear whether this pericalcarine grey matter atrophy is a visual system-specific phenomenon, or whether localised atrophy is also present in other cortical regions in these patients presenting with optic nerve demyelination. The pathophysiological substrates underlying early grey matter atrophy remain under debate. White matter lesions may be important, resulting in grey matter damage through secondary Wallerian or trans-synaptic tract degeneration. Alternatively, primary processes affecting grey matter may be more critical, either through a mechanism of focal cortical demyelination, or more widespread grey matter neurodegeneration, characterised by neuronal, dendritic and synaptic loss, occurring independent of white matter pathology.[5-8] Optic neuritis (ON) is a useful model with which to study these early relationships between white and grey matter damage, as both tissue types are clearly anatomically defined within the visual pathways, and $\mathrm{ON}$ is often the first symptom of MS.

The aims of this study were: (1) To determine whether pericalcarine atrophy predicts conversion to MS; (2) To investigate whether regional atrophy specifically affects visual cortex in patients with ON; (3) To investigate the potential causes of early pericalcarine atrophy using MRI. Mechanistically, our primary hypothesis was that pericalcarine atrophy may reflect a combination of visual white matter tract-specific damage (for example, from pre-existing optic radiation lesions), and early primary neurodegenerative changes in the visual grey matter, affecting those ON patients destined to develop 
MS. Our secondary hypothesis was that visual cortex might be more susceptible to damage than other cortical regions in patients presenting with ON. In order to investigate our primary hypothesis, we studied associations between early measures of tissue damage in the optic nerve, optic radiations and pericalcarine cortex at the time of presentation with clinically isolated $\mathrm{ON}$, and determined which of these were associated with developing MS a year later. For our secondary hypothesis, we compared regional cortical volumes in patients and controls, in frontal, parietal and temporal regions, to determine whether our previous finding of early regional atrophy was confined to pericalcarine cortex. In this manner, relationships between grey and white matter tissue damage early in MS were investigated.

\section{METHODS}

Patients with typical, acute, unilateral, clinically isolated $\mathrm{ON}$ were recruited. This was defined as a painful unilateral loss of vision, progressing over a few days to two weeks, [9] associated with signs of optic nerve dysfunction, but no additional neurological symptoms. The presence of a relative afferent pupillary defect was an inclusion criterion, in line with previous studies, such as the Optic Neuritis Treatment Trial.[10] Patients with a diagnosis of MS, bilateral ON, or other chronic neurological conditions were excluded. The presence of brain inflammatory lesions was not considered an exclusion criterion. Patients were invited for assessment within a month from symptom onset, and followed up after a year. Healthy age and sex matched controls were recruited over the same time period. All subjects gave informed written consent. The study was approved by the local Ethics Committee.

\section{Magnetic resonance imaging (MRI) of the visual pathway}

Structural imaging of the optic nerves and brain was performed at baseline in all subjects with a $1.5 \mathrm{~T}$ Signa Echospeed MRI system, with a maximum gradient strength of $33 \mathrm{mTm}^{-1}$ 


\section{Optic nerves}

(1) A coronal-oblique fast spin-echo sequence (TR 2300ms, TE 68ms, 2 excitations, echo train length 8 , matrix size $512 \times 384$, field of view (FOV) 24x18cm, 16 contiguous $3 \mathrm{~mm}$ slices) was acquired to calculate lesion length, which was determined by an experienced neuroradiologist (KM), blinded to image identity and side affected, by multiplying the number of consecutive slices of optic nerve returning abnormal signal by the slice thickness. The intra-observer coefficient of variation, which is the ratio of the standard deviation to the mean, multiplied by $100 \%$, was $2.8 \%$.

(2) Post triple-dose gadolinium-enhanced coronal-oblique fat-saturated T1-weighted spin-echo was acquired in patients at baseline (TR 600ms, TE 20ms, 1 excitation, matrix size 256x192, FOV $24 \times 18 \mathrm{~cm}, 16$ contiguous $3 \mathrm{~mm}$ slices).

(3) Coronal-oblique FLAIR imaging (TR 2500ms, TE 12.7ms, TI 995ms, 6 excitations, echo train length 6 , matrix size $512 \times 384$, FOV $24 \times 18 \mathrm{~cm}$, 16 contiguous $3 \mathrm{~mm}$ slices) was performed to obtain the optic nerve cross-sectional area, which was calculated by a single observer, blinded to image identity, from five contiguous slices anterior from the orbital apex,[11] using a semi-automated contouring technique.[12] The intra-observer reproducibility coefficient of variation was 4.6\%. In order to account for normal inter-individual variability, the ratio of affected to fellow nerve area was calculated.

\section{Optic radiations}

(1) Axial-oblique dual-echo fast spin-echo of the whole brain (TR 2000ms, TE 17ms/102ms, echo train length 8 , matrix size 256x256, FOV 24x18cm, 28 contiguous $5 \mathrm{~mm}$ slices) were acquired at baseline and 12 months, and used to calculate whole-brain and optic radiation lesion loads. Optic radiation lesions 
were identified by an experienced neuroradiologist, using standard anatomical landmarks. The intraobserver coefficient of variation was $2.6 \%$.

(2) Diffusion tensor imaging of the optic radiations and occipital lobe was obtained using an optimised single-shot, cardiac-gated, diffusion-weighted echo-planar imaging sequence (TR 10 RR 1113s, TE 82ms, 1 excitation, matrix size 96x96 (reconstructed to 128x128), FOV 22x22 $\mathrm{cm}^{2}$, in-plane resolution $2.3 \times 2.3 \mathrm{~mm}^{2}$ (reconstructed to $1.7 \times 1.7 \mathrm{~mm}^{2}$ ), 30 contiguous $2.3 \mathrm{~mm}$ slices, parallel to the ACPC line, diffusion gradients applied along 61 directions, [13] $b=1200 \mathrm{~s} / \mathrm{mm}^{2}$, optimised for white matter), and seven interleaved non-diffusion-weighted $b_{0}$ scans, acquisition time 10-15 minutes, depending on cardiac cycle. Head motion and eddy-current induced distortions were corrected and the diffusion tensor was then calculated on a pixel-by-pixel basis, using FSL tools (http://www.fmrib.ox.ac.uk/fsl/).

The optic radiations were reconstructed, using the probabilistic tractography algorithm provided by FSL (http://www.fmrib.ox.ac.uk/fsl/fdt/fdt_probtrackx.html).[14-15] The seed-points were defined in each Meyer's loop using functional MRI data, as described in detail elsewhere.[1] The mean fractional anisotropy (FA) within the tractography-derived tract was obtained for each side, in each subject.

\section{Visual cortex}

Three dimensional fast prepared spoiled gradient recall (3D-FSPGR) of the whole brain was acquired (TR 14.3ms, TE 5.1ms, 1 excitation, matrix size 256x128, FOV 31x31 cm, 156 contiguous $1.2 \mathrm{~mm}$ slices).

The images were analysed using FreeSurfer software (http://surfer .nmr.mgh.harvard.edu), in which they were reconstructed as 1x1x1mm axial images, and the brain extracted. The skull-strip was assessed visually in all cases, and manual correction was performed, if necessary, by a single observer, blinded to image identity. Fully automated cortical parcellation was then performed, and cortical volume 
and thickness estimates were obtained at baseline for pericalcarine cortex, middle temporal gyrus, precentral gyrus and post-central gyrus.[16-19]

An SPM5 segmentation-based methodology was used to extract whole-brain grey matter volume at baseline,[20] which was quantified using in-house software (http://www.nmrgroup.ion.ucl.ac.uk/atrophy/). The grey matter fraction was calculated by dividing the grey matter volume by the total intracranial volume (the sum of the grey matter, white matter and cerebrospinal fluid), for each patient, as in previous studies.[3]

\section{Statistical analysis}

Pericalcarine volume

Differences in baseline pericalcarine volume between patients with and without lesions, both throughout the whole brain and within the optic radiations, were calculated using two-sample, unpaired t-tests. Mean pericalcarine volumes are reported, summed for both hemispheres, together with the $95 \%$ confidence interval for each group.

\section{Development of $M S$}

The percentage of patients diagnosed with MS over the year of the study was calculated, using the revised McDonald criteria.[21] In addition, the number of patients with MRI activity (defined as typical white matter lesions insufficient to fulfill the criteria for MS) and no MRI activity (defined as a normal cranial MRI) are also reported, together with the number of patients with lesions involving the optic radiations. 
Associations between the development of MS at one year and baseline markers of tissue damage in the optic nerve, optic radiations, pericalcarine cortex and whole-brain white and grey matter were investigated. A multivariable regression approach was used, with clinical MS status at the end of the study as a binary dependent variable (0-no MS, 1-clinical diagnosis of MS). Separate models were specified, entering baseline data for each of the following as an independent variable: optic nerve lesion length, optic nerve area ratio, optic radiation lesion load and FA, pericalcarine cortical volume and thickness, whole-brain lesion load, grey matter volume and fraction, and cortical volume in the middle temporal, pre-central and post-central gyri. Associations were adjusted for age and gender.

A post-hoc analysis was performed to determine whether an association found between pericalcarine cortical volume and the development of MS was influenced by optic nerve and optic radiation damage measures, and whole-brain lesion load, by specifying a further multivariable regression model. In this model, MS status at 12 months was the dependent variable, baseline pericalcarine volume was always an independent variable, and each of the baseline optic nerve and radiation measures, and whole-brain lesion load, was entered, in turn, as an additional independent variable.

At 12 months, the patients were split into MS and non-MS groups. Two-sample unpaired t-tests were performed to investigate differences in baseline pericalcarine and whole-brain cortical measures between patients with and without MS at 12 months. Means and 95\% confidence intervals are reported.

\section{Baseline regional atrophy in other cortical areas}

Cortical regions-of-interest were specified a priori to represent each lobe of the brain: in addition to the pericalcarine region (occipital lobe), the middle temporal gyrus (temporal lobe), pre-central gyrus (frontal lobe), post-central gyrus (parietal lobe), whole-brain grey matter volume and whole-brain grey 
matter fraction were investigated. Differences in volume and thickness between patients and controls were investigated using two-sample, unpaired t-tests.

Associations between areas of damage within the visual pathway

The influence of optic nerve lesions, optic radiation lesions, and whole-brain lesion load on pericalcarine cortical atrophy at baseline was assessed by specifying separate multivariable regression models. Pericalcarine cortical volume and thickness were each specified, in turn, as the dependent variable. The following variables were each entered, in turn, as the independent variable: optic nerve fast spin-echo and gadolinium-enhanced lesion length, optic radiation lesion load and FA, and whole-brain lesion load.

\section{RESULTS}

Twenty-eight patients and ten healthy controls were recruited. Clinical and MRI outcome data at 12 months were available for $25 / 28$ patients. The median duration from symptom onset to baseline assessment was 22 days (range 7-34).

\section{Pericalcarine volume}

Pericalcarine volumes in ON patients were smaller in the presence of white matter lesions (whether in the whole brain or optic radiations) than in their absence (table 1). 
Table 1 Differences in baseline pericalcarine volumes between patients with and without brain lesions, and patients with and without optic radiation lesions

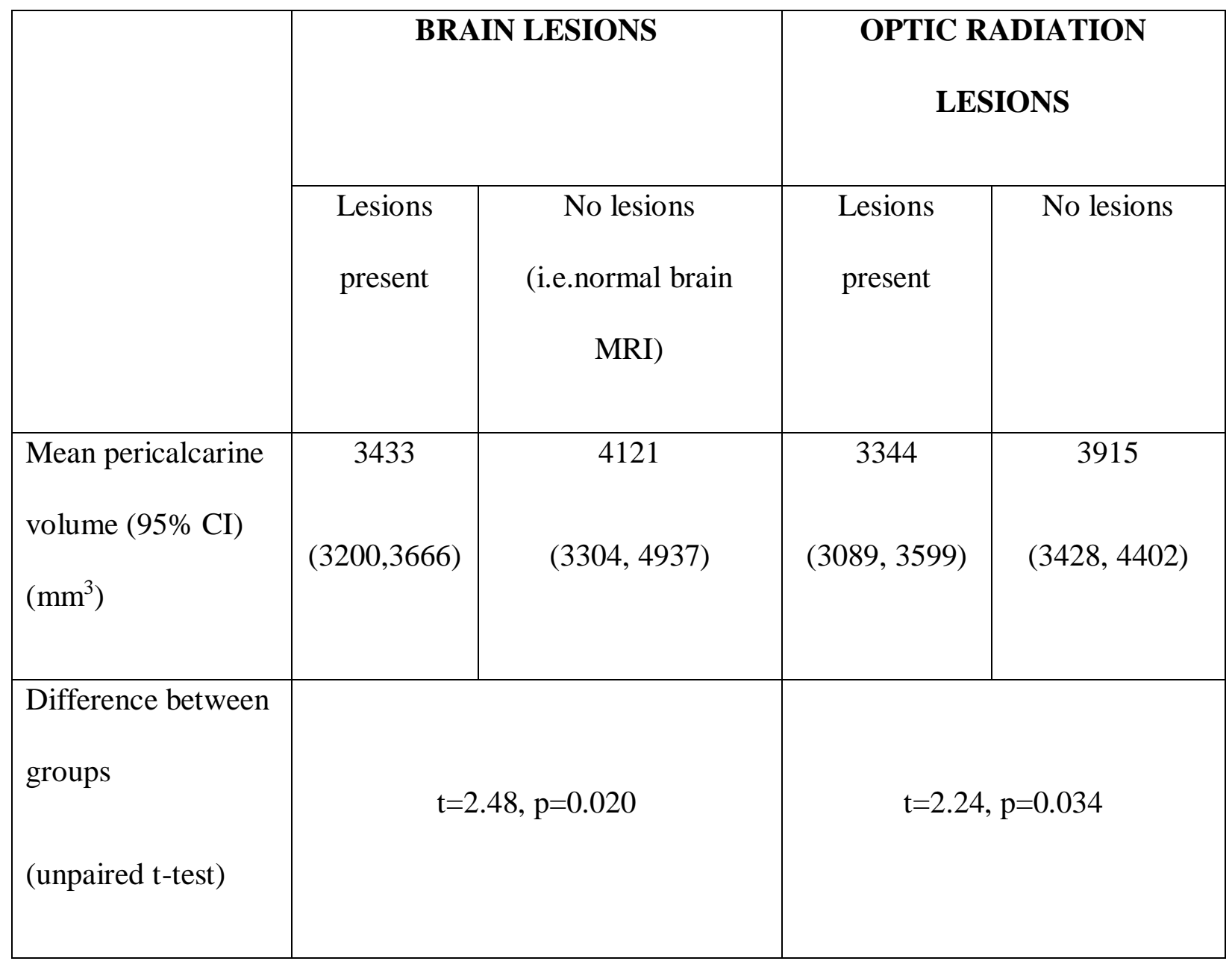

CI, confidence interval; $\mathrm{cm}$, centimetre.

\section{Development of MS}

Of the 28 patients, 12 developed MS, 9 had MRI activity and 6 had no activity by one year after ON onset. One patient with no activity at baseline was lost to follow-up. Fourteen patients had lesions in their optic radiations at baseline. 


\section{Associations between tissue damage and MS}

The following baseline variables were associated with the development of MS (table 2): greater optic radiation and whole-brain lesion load, shorter optic nerve lesion length, smaller pericalcarine cortical volume and thinner pericalcarine cortex. No significant associations were found between temporal, frontal and parietal cortical volumes and MS.

Table 2 Baseline variables associated with the development of MS at 12 months

\begin{tabular}{|c|c|c|}
\hline Structure & Parameter & P value (r) \\
\hline Optic nerve & FSE lesion length & $\mathbf{0 . 0 1 9}(\mathbf{- 0 . 4 7})$ \\
\hline & Gad lesion length & $\mathbf{0 . 0 1 3}(\mathbf{- 0 . 5 0 )}$ \\
\hline Optic radiation & Optic nerve area ratio & 0.175 \\
\hline Occipital cortex & Lesion load & $\mathbf{0 . 0 1 0}(\mathbf{0 . 5 1})$ \\
\hline & FA & 0.748 \\
\hline Whole brain & Pericalcarine volume & $\mathbf{0 . 0 3 2}(\mathbf{- 0 . 4 3})$ \\
\hline & Pericalcarine thickness & $\mathbf{0 . 0 4 9}(\mathbf{- 0 . 4 0})$ \\
\hline & Lesion load & $\mathbf{0 . 0 0 3}(\mathbf{0 . 5 7})$ \\
\hline & Grey matter volume & 0.765 \\
\hline
\end{tabular}

$\mathrm{P}$ values refer to the significance of each variable in the regression model. The partial correlation coefficient, $r$, is reported in parentheses for significant variables, which are in bold font.

FA, fractional anisotropy; FSE, fast spin-echo; Gad, gadolinium-enhanced scan.

All associations survived correction for age and gender, except pericalcarine cortical thickness, which retained borderline significance $(\mathrm{p}=0.065)$.

The association between baseline pericalcarine cortical volume and the development of MS one year after ON remained significant after adjusting for baseline optic radiation $\mathrm{FA}(\mathrm{p}=0.028)$, whole- 
brain grey matter volume ( $\mathrm{p}=0.038)$, and whole-brain grey matter fraction $(\mathrm{p}=0.015)$, but lost significance when the following baseline variables were entered into the model: optic nerve fast spinecho lesion length ( $\mathrm{p}$ value for pericalcarine volume in combined model 0.100 ), optic nerve gadoliniumenhanced lesion length $(\mathrm{p}=0.120)$, optic nerve area ratio $(\mathrm{p}=0.078)$, optic radiation lesion load $(\mathrm{p}=0.119)$ and whole-brain lesion load $(\mathrm{p}=0.122)$.

Means and $95 \%$ confidence intervals for each of the baseline pericalcarine cortical measures in the MS and non-MS groups are reported in table 3.

Table 3 Differences in baseline pericalcarine cortical measures between MS and non-MS groups at 12 months

\begin{tabular}{|c|c|c|c|}
\hline & MS & Non-MS & $\begin{array}{c}\text { Difference between } \\
\text { groups (p value for } \\
\text { unpaired t-test) }\end{array}$ \\
\hline Mean pericalcarine cortical volume & 3278 & 3907 & 0.032 \\
$\left(\mathrm{~mm}^{3}\right)$ & $(2968,3588)$ & $(3405,4409)$ & \\
\hline
\end{tabular}

There were no baseline differences in whole-brain grey matter volume and whole-brain grey matter fraction between patients who did and did not develop MS at one year $(p=0.765$ and $p=0.420$ respectively). 


\section{Baseline regional atrophy in other cortical areas}

There were no significant differences between patients and controls in middle temporal $(\mathrm{p}=0.296)$, precentral $(\mathrm{p}=0.100)$ or post-central $(\mathrm{p}=0.701)$ gyral volumes. There were no differences in whole-brain grey matter volume $(\mathrm{p}=0.222)$ or whole-brain grey matter fraction $(\mathrm{p}=0.607)$. In addition, no significant differences between patients and controls were found for cortical thickness in any region (outside visual cortex).

\section{Associations between areas of damage within the visual pathway}

There were no significant baseline associations between either of the pericalcarine MRI measures and optic nerve lesion length, optic radiation lesion load, optic radiation FA or whole-brain lesion load.

\section{DISCUSSION}

The most interesting finding of this study was the association between early pericalcarine cortical volume/thickness and the subsequent development of MS. In the sub-group of ON patients who developed MS over the following year, there was evidence for reduced grey matter in visual areas early after symptom onset. What could be the potential reasons for this? One possibility is the occurrence of secondary "downstream" degenerative effects resulting from the optic nerve lesion, or pre-existing lesions in the optic radiations. Against a hypothesis implicating the optic nerve lesion is the relatively early assessments of ON patients after symptom onset (range 7-34 days), perhaps too soon for quantifiable secondary degeneration to occur. Secondly, against either a hypothesis implicating the optic nerve lesion or optic radiation lesions, our observation that no markers of visual pathway damage 
(especially in these structures) were directly associated with pericalcarine MRI variables. However, these hypotheses cannot be entirely excluded, especially if the latter finding was insufficiently powered.

An alternative, independent hypothesis is that the observed pericalcarine cortical loss is a marker of a common, underlying disease process in patients who eventually develop MS. In support of this, the post-hoc analysis showed that the association between early pericalcarine volume/thickness and MS at one year was partially dependent on damage to the optic nerve, optic radiations and white matter lesion load in the whole brain, suggesting some shared variance. All these covariates may be markers for the evolution of MS pathology, and hence would naturally share some variance. Grey matter demyelination or primary neuroaxonal degeneration could explain why an aspect of pericalcarine volume loss is not explained by white matter damage, evidenced by the lack of direct associations with the lesion loads.

Our findings also suggest that visual grey matter loss is selectively predictive over other cortical regions for the development of MS. The reasons for this are unclear from this study. It is well recognised that the optic nerves and radiations are sites of predilection in MS. We selected a patient group with clinically isolated ON who all, by definition, had early white matter involvement. Therefore, it is possible that the visual grey matter shares characteristics with the visual white matter which render it especially vulnerable in this group. Possible explanations could include anatomical factors related to vascular supply, fibre orientation and adjacency to CSF spaces, or antigenic factors, specific to the neurons of the visual system.

Previous studies have identified progressive generalised cortical atrophy following clinically isolated syndromes in patients who develop MS.[2-3] However, atrophy does not appear to occur in those whose disease remains isolated,[3] even after 20 years.[22] In the early stages of MS, regional grey matter volume loss has been detected within the thalami[4] (from as early as four months after the initial event) and in other deep grey nuclei.[23] Following ON, optic radiation and pericalcarine 
abnormalities occur, identified from previous studies using quantitative diffusion MRI[24] and magnetisation transfer[25] measures, respectively; pericalcarine atrophy has also been previously reported.[26] The present cohort is, to our knowledge, the earliest that regional cortical atrophy has been reported following a clinically isolated syndrome. This pericalcarine atrophy was evident before detectable generalised grey matter volume loss, or regional atrophy in other cortical regions, and may therefore be a useful as a surrogate marker of neuroaxonal loss early in MS, at least in patients presenting with ON.

Another interesting observation from this study was that shorter acute lesions in the optic nerve were associated with the development of MS. Previous clinical studies have reported that severe optic nerve inflammation is relatively unusual in MS-related ON, although it does occur. Severe involvement is a "red flag" in ON, requiring investigations that consider alternative aetiologies, such as steroidresponsive optic neuritides.[9] No patient in this study was diagnosed with any condition other than MS over the course of the study, although it is possible that alternative diagnoses may emerge in the future. It therefore remains unclear why shorter lesions in ON were associated with MS in our cohort. In contrast, the strong associations between white matter lesions, both in the optic radiations and throughout the whole brain, and later development of MS were not surprising. It is well recognised that asymptomatic brain MRI lesions are associated with a much higher risk of subsequent MS,[27] and the optic radiations are common sites for incidental lesions.[28]

\section{Study limitations}

Cortical atrophy is defined in longitudinal studies as a reduction in grey matter volume over time. As this study was cross-sectional, although predictive, the term atrophy is used to describe a relatively smaller pericalcarine volume found in patients, compared with the control group. The size of the control 
group, and the inequality in size of the patient and control groups, are limitations of this study, as is the relatively large number of statistical comparisons, an issue which has been discussed in detail elsewhere.[29] It is also possible that methodological considerations could explain some of the observed findings; for example, cortical parcellation errors might not be uniform throughout the brain, resulting in regional differences in sensitivity to detect disease effects in the different lobes.

In conclusion, this study of patients with acute $\mathrm{ON}$ found an association between early pericalcarine cortical atrophy and subsequent conversion to MS at one year. Region-specific atrophy was not detectable at this early stage in other cortical regions, or throughout the whole brain. Therefore, both the grey and white matter of the visual system may be particularly susceptible to early damage in patients with ON who subsequently develop MS.

\section{ACKNOWLEDGEMENTS}

The authors thank the participants of the study, Dr G. Plant for clinical input, Dr. K. Miszkiel for neuroradiological input, and Drs. D. Altmann and C. Kallis for statistical advice. We thank the MS Society of Great Britain and Northern Ireland for their support of this study (Dr. T. Jenkins, grant 815/04) and for their support of the NMR Unit, including a recent programme grant which funded a new scanner. Part of this work was undertaken at UCLH/UCL which received a proportion of funding from the Department of Health's NIHR Biomedical Research Centres Funding Scheme. Dr. A. Toosy is supported by the Higher Education Funding Council for England. Dr. O. Ciccarelli is a Wellcome Trust Advanced Clinical Research Fellow. 


\section{REFERENCES}

1. Jenkins TM, Ciccarelli O, Toosy AT, et al. Dissecting structure-function interactions in acute optic neuritis to investigate neuroplasticity. Hum Brain Mapp 2010;31:276-86.

2. Dalton CM, Chard DT, Davies GR, et al. Early development of multiple sclerosis is associated with progressive grey matter atrophy in patients presenting with clinically isolated syndromes. Brain 2004;127:1101-07.

3. Fisher E, Lee JC, Nakamura K, et al. Gray matter atrophy in multiple sclerosis: a longitudinal study. Ann Neurol 2008;64:255-65.

4. Henry RG, Shieh M, Okuda DT, et al. Regional grey matter atrophy in clinically isolated syndromes at presentation. J Neurol Neurosurg Psychiatry 2008;79:1236-44.

5. Sepulcre J, Goni BS, Masdeu JC, et al. Contribution of white matter lesions to gray matter atrophy in multiple sclerosis. Arch Neurol 2009;66:173-79.

6. Chard D, Miller D. Grey matter pathology in clinically early multiple sclerosis: evidence from magnetic resonance imaging. J Neurol Sci 2009;282:5-11.

7. Antulov R, Carone DA, Bruce J, et al. Regionally distinct white matter lesions do not contribute to regional gray matter atrophy in patients with multiple sclerosis. J Neuroimaging Published Online First: April 132010 doi 10.1111/j.1552-6569.2010.00482.x

8. Bo L, Geurts JJ, van der Valk P, et al. Lack of correlation between cortical demyelination and white matter pathologic changes in multiple sclerosis. Arch Neurol 2007;64:76-80. 
9. Hickman SJ, Dalton CM, Miller DH, et al. Management of acute optic neuritis. Lancet 2002;360:1953-62.

10. Beck RW, Cleary PA, Anderson MM Jr, et al. A randomized, controlled trial of corticosteroids in the treatment of acute optic neuritis. The Optic Neuritis Study Group. N Engl J Med 1992;326:581-8.

11. Hickman SJ, Brex PA, Brierley CM, et al. Detection of optic nerve atrophy following a single episode of unilateral optic neuritis by MRI using a fat-saturated short-echo fast FLAIR sequence. Neuroradiology 2001;43:123-8.

12. Plummer DL. DispImage: A display and analysis tool for medical images. Rivista di Neuroradiologia 1992;5:489-95.

13. Cook PA, Symms M, Boulby PA, et al. Optimal acquisition orders of diffusion-weighted MRI measurements. J Magn Reson Imaging 2007;25:1051-8.

14. Behrens TE, Johansen-Berg H, Woolrich MW, et al. Non-invasive mapping of connections between human thalamus and cortex using diffusion imaging. Nat Neurosci 2003;6:750-7.

15. Behrens TE, Woolrich MW, Jenkinson M, et al. Characterization and propagation of uncertainty in diffusion-weighted MR imaging. Magn Reson Med 2003;50:1077-88.

16. Dale AM, Fischl B, Sereno MI. Cortical surface-based analysis. I. Segmentation and surface reconstruction. Neuroimage 1999;9:179-94.

17. Fischl B, Sereno MI, Dale AM. Cortical surface-based analysis. II: Inflation, flattening, and a surface-based coordinate system. Neuroimage 1999;9:195-207. 
18. Fischl B, Dale AM. Measuring the thickness of the human cerebral cortex from magnetic resonance images. Proc Natl Acad Sci USA 2000;97:11050-55.

19. Fischl B, van der KA, Destrieux C, et al. Automatically parcellating the human cerebral cortex. Cereb Cortex 2004;14:11-22.

20. Chard DT, Parker GJ, Griffin CM, et al. The reproducibility and sensitivity of brain tissue volume measurements derived from an SPM-based segmentation methodology. J Magn Reson Imaging 2002;15:259-67.

21. Polman CH, Reingold SC, Edan G, et al. Diagnostic criteria for multiple sclerosis: 2005 revisions to the "McDonald Criteria". Ann Neurol 2005;58:840-6.

22. Fisniku LK, Chard DT, Jackson JS, et al. Gray matter atrophy is related to long-term disability in multiple sclerosis. Ann Neurol 2008;64:247-54.

23. Audoin B, Zaaraoui W, Reuter F, et al. Atrophy mainly affects the limbic system and the deep grey matter at the first stage of multiple sclerosis. J Neurol Neurosurg Psychiatry 2010;81:690-5.

24. Ciccarelli O, Toosy AT, Hickman SJ, et al. Optic radiation changes after optic neuritis detected by tractography-based group mapping. Hum Brain Mapp 2005;25:308-16.

25. Audoin B, Fernando KT, Swanton JK, et al. Selective magnetization transfer ratio decrease in the visual cortex following optic neuritis. Brain 2006;129:1031-9.

26. Calabrese M, Atzori M, Bernardi V, et al. Cortical atrophy is relevant in multiple sclerosis at clinical onset. J Neurol 2007;254:1212-20. 
27. Optic Neuritis Study Group. Multiple sclerosis risk after optic neuritis: final optic neuritis treatment trial follow-up. Arch Neurol 2008;65:727-32.

28. Hornabrook RS, Miller DH, Newton MR, et al. Frequent involvement of the optic radiation in patients with acute isolated optic neuritis. Neurology 1992;42:77-9.

29. Jenkins TM, Toosy AT, Ciccarelli O, et al. Neuroplasticity predicts outcome of acute optic neuritis independent of tissue damage. Ann Neurol 2010;67:99-113. 